\title{
Clinical response to a lomustine/cytarabine-based chemotherapy protocol in a case of canine large granular lymphocyte $T$-cell lymphoma with spinal involvement
}

\author{
Elisabetta Treggiari ${ }^{1,2, *}$ and Lorna Provan Arrol $^{1}$ \\ ${ }^{l}$ Willows Veterinary Centre and Referral Service, Highlands Road, Solihull, B904NH, West Midlands, UK \\ ${ }^{2}$ Current Address: Centro Specialistico Veterinario, 20141, Milan, Italy
}

\begin{abstract}
A 7-year-old, female neutered cross-breed dog was referred to our institution with a history of progressive hind limb weakness, which then progressed to paraplegia. An MRI of the spine revealed severe meningeal infiltrate consistent with lymphoma involvement, located at the level of L2-L7 with concurrent lymph node enlargement and abnormal bone marrow. Abdominal ultrasonography also identified changes in the spleen and confirmed enlargement of the lumbar aortic lymph node. Cytology of lymph nodes and spleen confirmed a high-grade lymphoma with features of a large granular lymphocyte (LGL) variant; PCR for antigen receptor re-arrangements (PARR) was positive for a clonal T-cell receptor rearrangement. The dog was started on a chemotherapy protocol with lomustine and cytarabine incorporation and had a rapid improvement in neurological status. Chemotherapy was continued until relapse and rescue treatment used at that time. The dog was euthanased at the time of recurrence of neurological signs, 195 days since medical treatment was started. This case report suggests that combination chemotherapy may be of use when treating LGL lymphoma with spinal involvement and survival time may potentially exceed 6 months.
\end{abstract}

Keywords: Chemotherapy, Dog, Large granular lymphocyte, Lymphoma, Spinal.

\section{Introduction}

Spinal lymphoma is poorly described in dogs. The current literature is sparse, and only a few reports of dogs that underwent medical treatment exist. The first study to review the outcome of 37 spinal tumours (Levy et al., 1997) included 3 dogs with lymphoma, of which 2 were treated with radiotherapy and chemotherapy and survived 240 ad 730 days, respectively. However, no further details on the type of chemotherapy treatment used were available. In a single case report, one dog with lymphoma at the level of the brachial plexus was treated with lomustine followed by radiotherapy (RT) (Ueno et al., 2014); the dog showed clinical deterioration and died 53 days after starting treatment. Other case reports provide additional information on the location and immunohistochemistry (IHC) of the lesions identified on necropsy, where the T-cell immunophenotype appeared to be more common in dogs with spinal lymphoma (Rosin, 1982; Dallman and Saunders, 1986; Ortega and Castillo-Alcala, 2010). Magnetic resonance imaging (MRI) features of lymphoma have been described in the literature (Palus et al., 2012; Allett and Hecht, 2016) and, although this technique may help in the diagnostic process, cytology or histopathology are usually required for confirmation. Cerebrospinal fluid (CSF) analysis may also provide a diagnosis by the identification of abnormal lymphocytes (Vandevelde and Spano, 1977). A variant of large granular lymphocyte (LGL) lymphoma has been described in dogs in different anatomical locations, including the intestinal tract (Snead, 2007) and presumably the mediastinum (Ortiz et al., 2015); in one case treated with a multidrug chemotherapy protocol, the dog survived 28 days and was ultimately euthanased due to progression of clinical signs (Ortiz et al., 2015). Treatment options for localised lymphoma include surgery, RT and chemotherapy alone or in combination. In those cases affected by the multicentric form, where the disease appears to involve other organs (e.g. lymph nodes, internal organs and/or bone marrow), systemic treatment with cytotoxic chemotherapy is usually required, although the response and outcome are unclear. Additionally, to the authors' knowledge, no cases of LGL lymphoma with spinal involvement have been reported in the literature so far. The aim of this case report is to describe the outcome of a case of canine LGL lymphoma with spinal involvement and its response to a lomustine/cytarabinebased chemotherapy protocol.

\section{Case details}

A 7-year-old, female neutered cross-breed dog, was referred to our centre with a 2 week history of hindlimb weakness, which then progressed to paraplegia. No concurrent systemic signs were reported. Prior to referral to this centre, an MRI scan of the thoracic and lumbar spine was performed. The spinal cord and

*Corresponding Author: Elisabetta Treggiari. Willows Veterinary Centre and Referral Service, Highlands Road, Solihull, B90 4NH, UK. Tel.: +44 (0)121 7127070; Fax: +44 (0)121 7127071. Email: e.treggiari@ gmail.com 
meninges were grossly abnormal from the level of L1 caudally, with extensive swelling of the cord. The lesion in the spinal cord was hyper intense on $\mathrm{T} 2 \mathrm{~W}$ images. The meninges were thickened surrounding the spinal cord from L2-L7 causing circumferential compression of the spinal cord. The abnormal meninges were homogenous in appearance and intermediate in signal on $\mathrm{T} 2 \mathrm{~W}$ images. $\mathrm{T} 1 \mathrm{~W}$ post contrast images revealed a dense, homogenous enhancement of the abnormal meninges at the level of L4 (Fig. 1). Within the abdomen, a lesion consistent with enlargement of the right lumbar aortic lymph node was identified (Fig. 2). Due to the clinical suspicion of lymphoma, the case was referred to our institution for further investigations. At presentation, the dog was alert and in good body condition (BCS 5/9); her body weight was $28.6 \mathrm{~kg}$. Physical examination revealed no significant abnormalities except for a bilateral muscle atrophy affecting both pelvic limbs. Neurological examination revealed a normal mentation. The dog was paraplegic with deep pain sensation intact in the right pelvic limb but absent in the left pelvic limb. The segmental spinal reflexes were absent in the pelvic limbs and deep pain sensation was absent in the left and present in the right. The perineal reflex was reduced. The cutaneous trunci reflex was intact. Neurological examination of the thoracic limbs revealed normal voluntary movement, proprioception to be present and segmental spinal reflexes to be intact. Cranial nerve examination revealed no abnormalities. Spinal pain was evident on palpation of the lower lumbar spine. The neurological examination indicated a lesion affecting the L4-S3 segment of the spinal cord. The peripheral lymph nodes were normally palpable and rectal examination revealed no remarkable findings. The MRI scan was reviewed confirming the suspicion of lymphoma, with a lesion consistent with neoplastic infiltration at the level of L4 and extension to the right lumbar aortic lymph node. Abdominal ultrasonography was performed under sedation and identified splenomegaly with increased echogenicity and nodular lesions, with confirmed enlargement of the right lumbar aortic lymph node. The liver and the remainder of the internal lymph nodes and other organs were within normal limits. Aspirates of the right lumbar aortic lymph node, spleen and liver were collected, together with additional samples for flow cytometry collected from the splenic aspirates. Cytology was consistent with a high grade lymphoma with a large granular lymphocyte (LGL) morphology identified on samples of the lymph node (Fig. 3, 4) and spleen (Fig. 5); flow cytometry was not diagnostic due to the poor cell preservation, whilst PCR for antigen receptor gene rearrangements (PARR) was positive for a clonal T-cell receptor rearrangement, confirming a $\mathrm{T}$ - cell lymphoma.

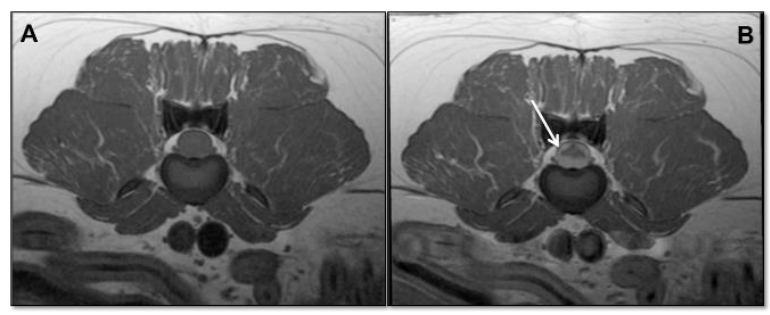

Fig. 1. MRI of the spine at the level of L4/L5. Transverse T1 $\mathrm{W}$ (A) and $\mathrm{T} 1 \mathrm{~W}$ post contrast (B) are shown. On the post contrast image there is dense, homogenous enhancement of the abnormal meninges (arrow) and, due to the severe meningeal thickening, there is circumferential compression of the spinal cord.

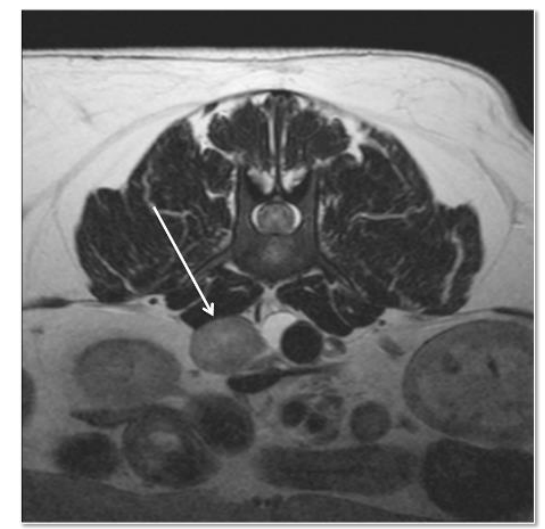

Fig. 2. Magnetic resonance imaging (MRI) study at the level of L3 on a T2W sequence, transverse view. Note the enlarged right lumbar aortic lymph node (arrow), which was later sampled and cytology found to be consistent with a large granular lymphocyte lymphoma.

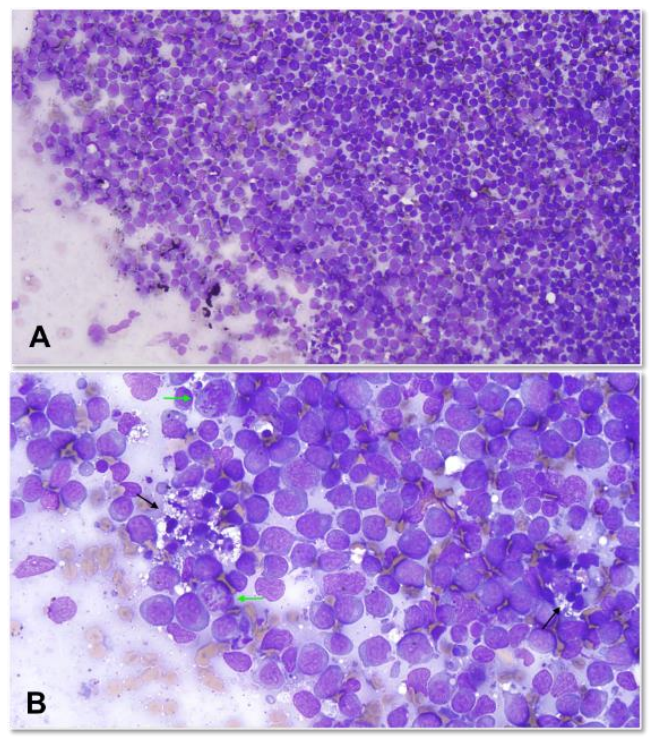

Fig. 3. Fine-needle aspirates from a lymph node of a dog with a large granular lymphocyte lymphoma. A, B: monomorphic population of large lymphoid cells. Mitoses are frequently observed (B: green arrows). Low numbers of tingible body macrophages are present (B: black arrow). Wright-Giemsa, 20X (A), 50X (B). 


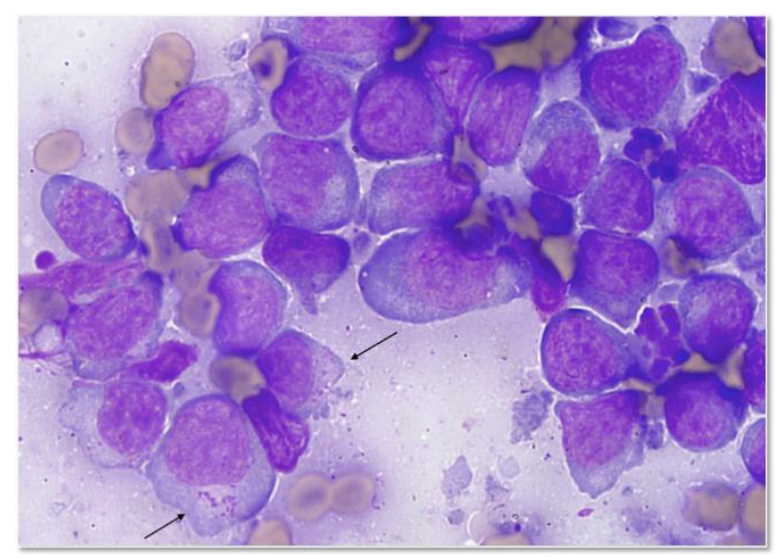

Fig. 4. Fine-needle aspirates from a lymph node of a dog with a large granular lymphocyte lymphoma. Monomorphic population of large lymphoid cells, some of which contain variable numbers of fine azurophilic intracytoplasmic granules (black arrows). Wright Giemsa, 100X.

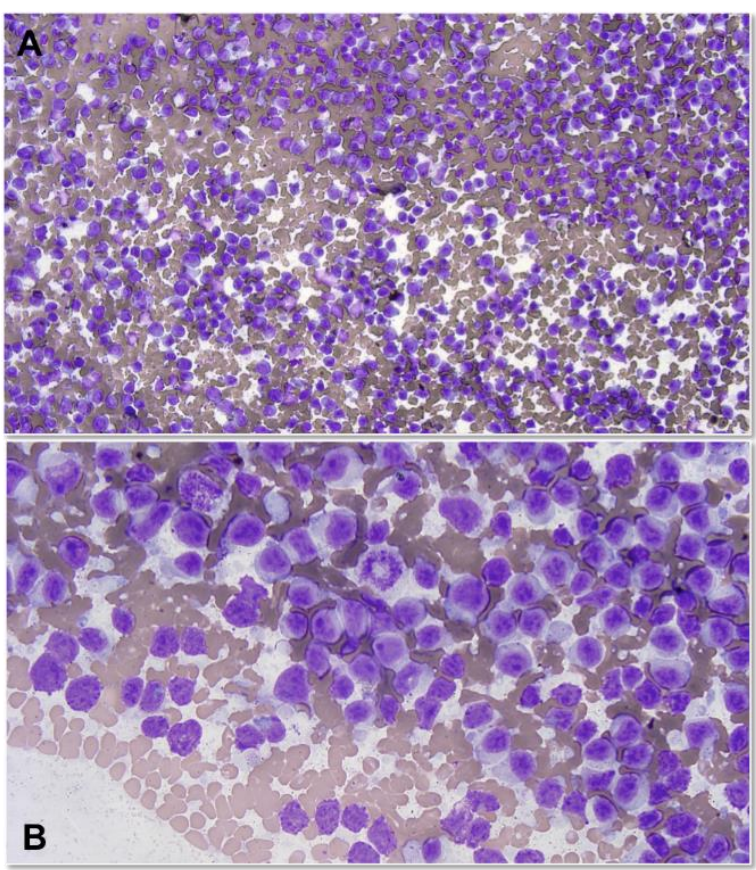

Fig. 5. Fine-needle aspirates from the spleen of a dog with LGL lymphoma (A, B). There are high numbers of monomorphic large lymphoid cells similar to those observed in the lymph node. Diff-Quick, (A) 20X, (B) 50X.

Given the evidence of multicentric disease and the abnormal changes at the level of the spinal cord and meninges visible on MRI, which were compatible with lymphoma, systemic chemotherapy was recommended. A haematology with a manual differential count and a biochemistry profile were performed, showing no presence of abnormal circulating lymphoid cells nor other major abnormalities. A multidrug chemotherapy protocol was discussed with the owners (Table 1). The patient initially received $10,000 \mathrm{IU} / \mathrm{m}^{2}$ of Lasparaginase intramuscularly (IM), followed by dexamethasone $0.3 \mathrm{mg} / \mathrm{kg}$ intravenously (IV). After 24 hours, the dog regained deep pain sensation in the left pelvic limb, and treatment was continued with cytarabine $150 \mathrm{mg} / \mathrm{m}^{2}$ q 12 hours subcutaneously (SC) for the following 48 hours. The dog's neurological condition continued to improve and the patient regained good voluntary movement after 5 days of therapy and intact segmental spinal reflexes in the pelvic limbs. Additional chemotherapy was administered at that stage prior to discharge, consisting of vincristine as an IV bolus at $0.5 \mathrm{mg} / \mathrm{m}^{2}$. A reduced dose was used due to an asymptomatic non-febrile neutropenia (Veterinary Cooperative Oncology group- VCOG grade I) and earlier administration than planned, due to logistical reasons. The dog was discharged on oral prednisolone and omeprazole and a dose reduction of corticosteroids was applied as per protocol (Table 1). She represented a week later to continue chemotherapy with oral lomustine at $60 \mathrm{mg} / \mathrm{m}^{2}$. At that stage, the dog's gait was ambulatory paraparetic. The dose-limiting side effect was an afebrile VCOG grade III neutropenia, one-week post lomustine administration; a 5 days course of a broad-spectrum, oral antibiotic (potentiated amoxicillin) was prescribed and the patient recovered uneventfully. Due to the documented neutropenic events, dose reductions were applied for the remainder of the protocol: vincristine was administered at a reduced dose of $0.5 \mathrm{mg} / \mathrm{m}^{2} \mathrm{IV}$ and lomustine at 50 $\mathrm{mg} / \mathrm{m}^{2} \mathrm{PO}$. Cytarabine was continued at $150 \mathrm{mg} / \mathrm{m}^{2} \mathrm{SC}$ q 12 hours for 48 hours. The dog experienced neurological deterioration 152 days after treatment was started, formally at week 19 of the protocol. Neurological examination revealed ambulatory paraparesis with mild proprioceptive deficits in the pelvic limbs and reduced segmental spinal reflexes; an L4-S3 neurolocalisation was suspected. At that stage, she received L-asparaginase SC to try and consolidate the response together with the planned cytarabine treatment. She represented 2 weeks later at which point the gait had deteriorated and, although still ambulatory, the dog had marked proprioceptive deficits in both pelvic limbs. An anthracycline-based rescue protocol was offered at that stage but, due to a VCOG grade III thrombocytopenia, inappetance and diarrhoea, it was elected to start prednisolone at $40 \mathrm{mg} / \mathrm{m}^{2}$ SID PO only. A haematology with a manual differential count was reassessed 7 days later and the thrombocytopenia found to have resolved; the dog's appetite was back to normal and her demeanour had also improved. Epirubicin as single agent was started at that stage; this was administered as a slow IV infusion over 20 minutes at a standard dose of $30 \mathrm{mg} / \mathrm{m}^{2}$ diluted with $0.9 \% \mathrm{NaCl}$, following administration of maropitant at $1 \mathrm{mg} / \mathrm{kg}$ IV. Additional oral maropitant was prescribed for use at home for an extra 4 days at a standard dose of $2 \mathrm{mg} / \mathrm{kg}$ PO q 24 hours. 
Table 1. Chemotherapy protocol with lomustine and cytarabine incorporation.

\begin{tabular}{|c|c|c|c|c|c|c|c|c|c|c|c|c|c|c|c|c|c|}
\hline \multirow{2}{*}{ Chemotherapy } & \multicolumn{17}{|c|}{ Week } \\
\hline & 1 & 2 & 3 & 5 & 6 & 7 & 8 & 9 & 10 & 11 & 13 & 15 & 17 & 19 & 21 & 23 & 25 \\
\hline $\begin{array}{l}\text { L-asparaginase } \\
\left(10,000 \mathrm{IU} / \mathrm{m}^{2}\right) \mathrm{IM} \\
\text { or SC }\end{array}$ & $*$ & & & & & & & & & & & & & & & & \\
\hline $\begin{array}{l}\text { Vincristine } \\
\left(0.5-0.7 \mathrm{mg} / \mathrm{m}^{2} \mathrm{IV}\right)\end{array}$ & & $*$ & & & $*$ & & & & $*$ & & & $*$ & & & $*$ & & \\
\hline $\begin{array}{l}\text { Lomustine } \\
{[\mathrm{CCNU}](50-60} \\
\left.\mathrm{mg} / \mathrm{m}^{2} \mathrm{PO}\right)\end{array}$ & & & $*$ & & & $*$ & & & & $*$ & & & $*$ & & & $*$ & \\
\hline $\begin{array}{l}\text { Cytarabine } \\
\left(150 \mathrm{mg} / \mathrm{mg}^{2} \mathrm{SC} \mathrm{q}\right. \\
12 \text { hours for } 48 \\
\text { hours; total } 600 \\
\left.\mathrm{mg} / \mathrm{m}^{2}\right)\end{array}$ & $*$ & & & $*$ & & & & $*$ & & & $*$ & & & $*$ & & & $*$ \\
\hline $\begin{array}{l}\text { Prednisolone } \\
\left(40 \mathrm{mg} / \mathrm{m}^{2} \text { week } 1 \text {; }\right. \\
30 \mathrm{mg} / \mathrm{m}^{2} \text { week } 2 ; \\
20 \mathrm{mg} / \mathrm{m}^{2} \text { week } 3 ; \\
\left.10 \mathrm{mg} / \mathrm{m}^{2} \text { week } 4\right)\end{array}$ & $*$ & * & $*$ & $*$ & & & & & & & & & & & & & \\
\hline
\end{tabular}

The dog experienced a partial clinical response (PR) and no adverse events a week after chemotherapy. However, 14 days since epirubicin was administered, the neurological signs recurred in the form of progressive weakness on the hind limbs and, although further chemotherapy was offered and discussed at that time, the owner elected to discontinue treatment and administer prednisolone only at a dose of $40 \mathrm{mg} / \mathrm{m}^{2}$ PO q 24 hours. The patient eventually developed paraplegia 7 days later and she was euthanased due to severe clinical deterioration. The overall survival was 195 days since chemotherapy treatment was started.

\section{Discussion}

This case report describes response to chemotherapy in a case of LGL lymphoma with spinal involvement and infiltration of the right lumbar aortic lymph node and the spleen, where the dog initially presented with paraplegia. To the author's knowledge, this is the first report of a LGL lymphoma with spinal involvement, although reports of lymphomas of similar morphology but with a different anatomical location exist in domestic dogs (Snead, 2007; Ortiz et al., 2015). When comparing response to treatment and survival to similar cases, our patient became ambulatory without support soon after starting chemotherapy and the overall survival of 195 days was longer than previously reported in a dog treated with lomustine and RT (Ueno et al., 2014) and another dog that received a lomustinebased combination protocol (Ortiz et al., 2015).

Chemotherapy was overall well tolerated and the patient did not have to be hospitalised due to AEs. The only reported AEs were mild to moderate haematological toxicoses, although they were not related to concurrent clinical signs. However, due to treatment delays especially within the first few weeks of treatment, we cannot rule out that this may have affected the overall survival and response. Although it is often said that unnecessary dose reductions may influence the response in dogs with lymphoma, these appeared to be necessary not to incur into further treatment delays and, as such, reduced doses of chemotherapy would be recommended from the beginning if a dose-intense protocol like the one used in this case report is employed.

The chemotherapy drugs included in this protocol were chosen based on their documented ability of crossing the blood brain barrier in dogs (Scott-Moncrieff et al., 1991), due to the presence of spinal involvement. Cytarabine was used as a 48 hours SC injection rather than a continuous infusion, due to the dog's temperament. However, a recent study has shown that plasma levels of this drug are affected by its route of administration and its steady state and excretion appear to be more rapid through the SC route (Crook et al., 2013). As such, a cytarabine infusion can still be considered in a similar case. It is unclear if a different multidrug protocol (e.g. CHOP, where C stays for cyclophosphamide, $\mathrm{H}$ for adryamycin, $\mathrm{O}$ for vincristine and $\mathrm{P}$ for prednisone) would have resulted in a similar outcome, and clearly larger studies would be needed to assess the efficacy of different chemotherapy protocols in treating lymphomas with spinal involvement. However, the rapid improvement in clinical signs and resolution of paraplegia was encouraging and the same protocol was therefore continued until the time of relapse. L-asparaginase was used due to its rapid effect, 
tolerability and fewer myelosuppressive events. Previous studies have not shown a real survival advantage when this drug was used at the time of induction in patients with multicentric lymphoma that were undergoing a CHOP protocol (MacDonald et al., 2005) and so it may have not affected the overall response. Also, two different routes of administration (IM and SC) were used, though in a study on dogs with multicentric lymphoma receiving doxorubicin, the IM route appeared to be associated with a longer response, duration of first remission and survival time (Valerius et al., 1999). In this case, the authors elected not to collect a CSF sample or sampling the spinal lesion due to the possibility of collecting a non diagnostic sample and potential risks associated with the procedure; instead, they elected to perform ultrasound guided samples of more accessible organs (lymph node and spleen), which turned out to be diagnostic and were consistent with the MRI findings. Cytology is considered a reliable method of diagnosing lymphoma and PARR has been shown to have an agreement with flow cytometry and immunohistochemistry of $97 \%$ for B-cell and $93 \%$ for T-cell lymphomas in dogs (Lana et al., 2006). Unfortunately, the sample collected in our case had insufficient cellularity to perform a flow cytometric analysis, although its validity has been described in a previous case report of LGL lymphoma (Ortiz et al., 2015). A repeat MRI was not performed to confirm remission status and therefore the response assessment was purely clinical and based on the neurological examination at the time of each follow-up revisit. This was mainly due to financial reasons but also due to the likelihood of detecting recurrence based on progression of clinical signs. Ultimately, a single agent anthracycline was chosen to treat signs of relapse given it was not included in the original protocol. The use of doxorubicin alone or in combination has been previously described to treat canine patients with relapsed lymphoma (Calvert and Leifer, 1981; Lori et al., 2010) and epirubicin has been used in a multidrug protocol to treat dogs with naïve lymphoma (Elliott $e t$ al., 2013). No other rescue protocols or consolidation of the above were tried as the owner had decided to discontinue treatment, but the authors cannot exclude that further chemotherapy may have resulted in prolonged remission and survival times, and can therefore be considered in a similar case.

In conclusion, this case report suggests that a protocol with lomustine and cytarabine incorporation may result in temporary resolution of clinical signs in dogs with LGL lymphoma and spinal involvement and possibly prolong survival when compared to similar cases described in the literature. The LGL subtype in this anatomical location may have a better prognosis when treated.

\section{Acknowledgements}

The authors would like to acknowledge Dr. Paola Monti, Dick White Referrals, Clinical Pathology Service, Six Mile Bottoms, UK, for providing the cytology pictures and their description; Dr. Luis Mesquita, Willows Veterinary Centre and Referral Service, Diagnostic Imaging Service, Solihull, UK, for providing the MRI pictures.

\section{Conflict of interest}

The authors declare that there is no conflict of interests.

\section{References}

Allett, B. and Hecht, S. 2016. Magnetic resonance imaging findings in the spine of six dogs diagnosed with lymphoma. Vet. Radiol. Ultrasound 57(2), 154-161.

Calvert, C.A. and Leifer, C.E. 1981. Doxorubicin for treatment of canine lymphosarcoma after development of resistance to combination chemotherapy. J. Am. Vet. Med. Assoc. 179(10), 1011-1012.

Crook, K.I., Early, P.J., Messenger, K.M., Munana, K.R., Gallagher, R. and Papich, M.G. 2013. The pharmacokinetics of cytarabine in dogs when administered via subcutaneous and continuous intravenous infusion routes. J. Vet. Pharmacol. Ther. 36, 408-411.

Dallman, M.J. and Saunders, G.K. 1986. Primary spinal cord lymphosarcoma in a dog. J. Am. Vet. Med. Assoc. 189, 1348-1349.

Elliott, J.W., Cripps, P., Marrington, A.M., Grant, I.A. and Blackwood, L. 2013. Epirubicin as part of a multi-agent chemotherapy protocol for canine lymphoma. Vet. Comp. Oncol. 11, 185-198.

Lana, S.E., Jackson, T.L., Burnett, R.C., Morley, P.S. and Avery, A.C. 2006. Utility of polymerase chain reaction for analysis of antigen receptor rearrangement in staging and predicting prognosis in dogs with lymphoma. J. Vet. Intern. Med. 20, 329-334.

Levy, M.S., Kapatkin, A.S., Patnaik, A.K., Mauldin, G.N. and Mauldin, G.E. 1997. Spinal tumors in 37 dogs: clinical outcome and long-term survival (1987-1994). J. Am. Anim. Hosp. Assoc. 33(4), 307-312.

Lori, J.C., Stein, T.J. and Thamm, D.H. 2010. Doxorubicin and cyclophosphamide for the treatment of canine lymphoma: a randomized, placebo-controlled study. Vet. Comp. Oncol. 8, 188-195.

MacDonald, V.S., Thamm, D.H., Kurzman, I.D., Turek, M.M. and Vail, D.M. 2005. Does Lasparaginase influence efficacy or toxicity when added to a standard CHOP protocol for dogs with lymphoma? J. Vet. Intern. Med. 19, 732-736. 
Ortega, M. and Castillo-Alcala, F. 2010. Hind-limb paresis in a dog with paralumbar solitary $\mathrm{T}$-cell lymphoma. Can. Vet. J. 51, 480-484.

Ortiz, A.L., Carvalho, S., Leo, C., Riondato, F., Archer, J. and Cian, F. 2015. Gamma delta T-cell large granular lymphocyte lymphoma in a dog. Vet. Clin. Pathol. 44, 442-447.

Palus, V., Volk, H.A., Lamb, C.R., Targett, M.P. and Cherubini, G.B. 2012. MRI features of CNS lymphoma in dogs and cats. Vet. Radiol. Ultrasound 53, 44-49.

Rosin, A. 1982. Neurologic diseases associated with lymphosarcoma in ten dogs. J. Am. Vet. Med. Assoc. 181, 50-53.

Scott-Moncrieff, J.C., Chan, T.C., Samuels, M.L., Cook, J.R., Coppoc, G.L., DeNicola, D.B. and Richardson, R.C. 1991. Plasma and cerebrospinal fluid pharmacokinetics of cytosine arabinoside in dogs. Cancer Chemother. Pharmacol. 29, 13-18.

Snead, E.C. 2007. Large granular intestinal lymphosarcoma and leukemia in a dog. Can. Vet. J. 48, 848-851.

Ueno, H., Miyoshi, K., Fukui, S., Kondo, Y., Matsuda, K. and Uchide, T. 2014. Extranodal lymphoma with peripheral nervous system involvement in a dog. $\mathrm{J}$. Vet. Med. Sci. 76, 723-727.

Valerius, K.D., Ogilvie, G.K., Fettman, M.J., Walton, J.A., Richardson, K., Powers, B.E., McNiel, E.A. and Rogers, Q.R. 1999. Comparison of the effects of asparaginase administered subcutaneously versus intramuscularly for treatment of multicentric lymphoma in dogs receiving doxorubicin. J. Am. Vet. Med. Assoc. 214, 353-356.

Vandevelde, M. and Spano, J.S. 1977. Cerebrospinal fluid cytology in canine neurologic disease. Am. J. Vet. Res. 38, 1827-1832. 